\title{
A NONLINEAR DIFFUSION EQUATION ARISING IN THE STUDY OF SOFT TISSUE*
}

\author{
By \\ MARK H. HOLMES \\ Rensselaer Polytechnic Institute
}

\begin{abstract}
A nonlinear diffusion equation which describes the deformation of a soft fluid-filled tissue is studied. The nonlinearity in the problem arises from the permeability of the elastic phase which for a number of tissues, such as articular cartilage, is strongly dependent on the strain. Moreover, for most tissues the exact dependence is not known, and so the functional dependence of the permeability on the strain is not determined until after the problem is solved. The approach uses perturbation methods for the diffusive boundary layers that occur in the problem and similarity solutions to solve the reduced problems. Once the solution is obtained, the permeability function is determined and some of the limitations of the present model of soft tissue are discussed.
\end{abstract}

Introduction. The study of the flow of a fluid through a porous material has a rather long and productive history. The major field of application has been in soil mechanics, although this is by no means the only one. For example, over the last few years the theory for a porous flow has been used in the study of the deformation of soft tissue. The principal application has been to articular cartilage, but it appears that the models of a number of other tissues, such as in muscle, in the heart, and in the study of the arterial wall, should incorporate many of the same ideas.

Structurally, articular cartilage consists of a deformable porous solid that is filled with fluid. Consequently, in studying the dynamical behavior of cartilage it is necessary to account for the deformation of the solid and how it interacts with the motion of the fluid. One of the complicating features in doing this involves the microstructure of the cartilage. The pores are of such a size that the permeability of the solid matrix changes significantly as the tissue deforms. In other words, the permeability depends on the strain tensor of the solid matrix. The only theoretical analysis on the resulting nonlinear diffusion problem has been by Lai et al. [4] who use successive approximations and numerical methods in solving the problem. The drawback to this approach is that it is difficult to see analytically how the deformation depends on the variable permeability or any other component of the

\footnotetext{
* Received February 23, 1982. The author would like to thank Dr. V. C. Mow and Dr. W. M. Lai for their enlightening discussions about articular cartilage, and John Schoonbeck for his experimental results. This work was supported by the National Science Foundation Grant MCS81-02129 and CME79-19524.
} 
structure of the tissue. This is important since the exact functional dependence of the permeability on the strain is still not completely resolved. For this reason the problem to be considered here is, in effect, an inverse problem since the coefficient function is unknown until after the solution is obtained.

It is the purpose of this article to analyze the diffusion in a soft porous medium where the permeability is an arbitrary function of the strain. The motion is unidirectional and the boundary conditions to be used correspond to the tissue being under compression. Besides being able to say how the tissue deforms as it is compressed, it will also be shown how the permeability function can be determined for any given tissue.

As a final comment it should be pointed out that although the problem to be discussed arises in studying the mechanics of articular cartilage, the analysis can be applied to a number of other diffusion problems. For example, the ideas can be applied in a straightforward manner to other problems involving a variable diffusivity, as the nonlinear extensions of Fick's law discussed in Shi et al. [6].

Formulation and scaling of model. The equations of motion for a deformable porous medium can be obtained using the mixture theories of Craine et al. [2] and Bowen [1]. In what follows we are interested in the case when the solid phase is an incompressible, elastic, isotropic porous body and the interstitial fluid is inviscid and incompressible. The deformations and velocities are assumed to be such that the stress-strain relationships for the solid are linear. Also, the densities and permeability are such that the inertia of both the solid and fluid components can be ignored. The bulk viscosity of the fluid phase is neglected, although the viscosity of the fluid does contribute implicitly through the diffusive drag force, which is obtained, essentially, from Darcy's law. That is to say, it is assumed that the diffusive drag is a linear function of the relative velocity between the solid and fluid.

With these assumptions, the equations of motion are

$$
\begin{aligned}
\nabla \cdot\left(\phi^{s} \vec{v}^{s}+\phi^{f} \vec{v}^{f}\right) & =0, \\
\nabla \cdot \sigma^{s}-K^{*}\left(\vec{v}^{s}-\vec{v}^{f}\right) & =\overrightarrow{0}, \\
\nabla \cdot \sigma^{f}+K^{*}\left(\vec{v}^{s}-\vec{v}^{f}\right) & =\overrightarrow{0},
\end{aligned}
$$

where $\phi^{s}$ and $\phi^{f}$ represent the volume fraction of the solid and fluid, respectively. Also,

$$
\boldsymbol{\sigma}^{f}=-\boldsymbol{\phi}^{f} p I
$$

and

$$
\sigma^{s}=-\phi^{s} p I+\lambda^{s} \operatorname{tr}(E) I+2 \mu^{s} E,
$$

where $p$ is a pressure and $E$ is the infinitesimal stain tensor for the solid phase. The coefficients $\lambda^{s}$ and $\mu^{s}$, which are the elastic moduli for the solid phase in the mixture, are assumed to be constant. The first of these equations is the continuity equation for the biphasic material [5]. The diffusive drag coefficient $K^{*}$ in the momentum equations is related to the permeability $k^{*}$ as follows [Lai and Mow, 3]:

$$
K^{*}=\left(\phi^{f}\right)^{2} / k^{*} \text {. }
$$


From this, and since the solid matrix is deformable, it is assumed that $K^{*}$ is a function of the first strain invariant for the solid phase [4].

The problem to be considered here comes from the one-dimensional motion that is obtained from (1). In particular, the tissue is taken to occupy the region $0 \leqslant z^{*} \leqslant h$ and the motion is entirely in the $z^{*}$ direction. The boundary $z^{*}=h$ is a fixed rigid impermeable surface, and on the surface $z^{*}=0$ the displacement of the solid phase is prescribed. Physically, this corresponds to compressing the tissue with a rigid porous filter at $z^{*}=0$ which, at the same time, permits the fluid to exude unimpaired from the tissue through the filter.

With these assumptions the problem reduces to solving the following diffusion equation for the deformation $u^{*}\left(z^{*}, t^{*}\right)$ of the solid phase:

$$
\lambda \partial_{z^{*}}^{2} u^{*}=K^{*}\left(\partial_{z^{*}} u^{*}\right) \partial_{t^{*}} u^{*}, \quad 0<z^{*}<h,
$$

where $\lambda=\left(\phi^{f}\right)^{2}\left(\lambda^{s}+2 \mu^{s}\right)$ is a material constant. The boundary and initial conditions are

$$
\begin{aligned}
u^{*}\left(0, t^{*}\right) & =u_{0}^{*} g\left(\frac{t^{*}}{t_{0}}\right), \quad 0 \leqslant t^{*} \leqslant t_{0}, \\
& =u_{0}^{*}, \quad t_{0}<t^{*},
\end{aligned}
$$

and

$$
u^{*}\left(h, t^{*}\right)=u^{*}\left(z^{*}, 0\right)=0 .
$$

The asterisks are used here to distinguish the variables from their dimensionless counterparts introduced below.

The forcing function $g(\cdot)$ is a known analytic function and is such that $g(0)=0$ and $g(1)=1$. Therefore, $(3 \mathrm{~b})$ corresponds to compactifying the solid up to time $t_{0}$, after which the displacement is held constant at $u_{0}^{*}$. As for $K^{*}$, since both phases of the tissue are incompressible, it is assumed that

$$
\begin{aligned}
K^{*}(s) & >0, \quad \forall s \leqslant 0, \\
\frac{d}{d s} K^{*}(s) & \leqslant 0, \quad \forall s \leqslant 0 .
\end{aligned}
$$

It is also assumed that $K^{*}$ is a smooth function of the compressive strain. However, aside from these assumptions, the diffusive drag coefficient $K^{*}$ is arbitrary.

The problem is scaled by letting $z^{*}=h z, t^{*}=t_{0} t$, and $u^{*}=u_{0}^{*} u(z, t)$. In this case (3) takes the form

$$
R^{2} \partial_{z}^{2} u=K\left(\varepsilon \partial_{z} u\right) \partial_{t} u, \quad 0<z<1,
$$

where

$$
\begin{aligned}
& u(0, t)=g(t), \quad 0 \leqslant t \leqslant 1, \\
& u(1, t)=u(z, 0)=0 .
\end{aligned}
$$

Also,

$$
\varepsilon=u_{0} / h, \quad R^{2}=\lambda t_{0} / h^{2} K^{*}(0),
$$


and

$$
K\left(\varepsilon \partial_{z} u\right)=K^{*}\left(\varepsilon \partial_{z} u\right) / K^{*}(0) .
$$

In (4b) the boundary condition for $t>1$ is not included as the problem is going to be solved only for $0 \leqslant t \leqslant 1$. However, the analysis extends in a relatively straightforward manner to include this later time interval.

The parameter $\varepsilon$ represents the equilibrium strain and it is assumed to be relatively small. As for $R$, in the case of linear diffusion $(K \equiv 1)$ it is a measure of the distance the disturbance has effectively propagated into the medium after time $t_{0}$ as compared to the thickness $h$. By changing $t_{0}$ it is possible for $R$ to vary appreciably. For articular cartilage, $R$ can be anywhere between $10^{-4}$ and $10^{2}$. It is how the deformation changes as $R$ varies over such a range of values that is considered here.

The results of the analysis to follow are usually compared with experiment by using the stress measured at the surface $z=0$. With the assumed linear stress-strain law, the stress at the surface is

$$
\sigma_{0}^{*}=\left(\lambda^{2}+2 \mu^{s}\right) \partial_{z^{*}} u^{*}\left(0, t^{*}\right) .
$$

Normalizing this with the equilibrium stress $\sigma_{\infty}^{*}=-\varepsilon\left(\lambda^{s}+2 \mu^{s}\right)$, we have that

$$
\sigma_{0}=-\partial_{z} u(0, t) \text {. }
$$

In the next three sections the various limits associated with $R$ are developed. The application and implications of these results are presented in the last section.

Slow rates of compression. The simplest limit to consider is when $R>>1$ for $\varepsilon>0$ fixed. The appropriate expansion in this case is

$$
u(z, t) \sim u_{0}(z, t)+u_{1}(z, t) / R^{2}+\ldots
$$

Substituting this into (4) and solving the reduced problems, one finds that

$$
u_{0}(z, t)=g(t)(1-z)
$$

and

$$
u_{1}(z, t)=-\frac{1}{6} z(z-1)(z-2) g^{\prime}(t) K[-\varepsilon g(t)] .
$$

The surface stress $(6)$ is therefore given as

$$
\sigma_{0} \sim g(t)+g^{\prime}(t) K[-\varepsilon g(t)] / 3 R^{2} .
$$

The complicating feature of this limit is that it is singular in $t$. However, this can be handled by introducing the initial layer with coordinate $\tau=R^{2} t$. In this case

$$
\partial_{z}^{2} u=K\left(\varepsilon \partial_{z} u\right) \partial_{\tau} u,
$$

where

$$
u(0, \tau)=g\left(\tau / R^{2}\right) .
$$

The expansion of $u$ in this region is

$$
u \sim g\left(1 / R^{2}\right)\left[U_{0}(z, \tau)+\ldots\right] .
$$


From the analyticity of $g$, for large $R$,

$$
g\left(\tau / R^{2}\right)=g_{0}\left(\tau / R^{2}\right)^{n}+O\left(1 / R^{2 n+2}\right),
$$

where $n \in \mathbb{Z}^{+}$and $g_{0}$ is a nonzero constant. So, introducing (10) into (9) and solving the $O(1)$ problem, one finds that

$$
U_{0}(z, \tau)=\sum_{k=0}^{n} \tau^{n-k} p_{k}(z)+V_{0}(z, \tau)
$$

where

$$
p_{0}(z)=g_{0}(1-z)
$$

and

$$
p_{k}^{\prime \prime}(z)=p_{k-1}(z), \quad k=1, \ldots, n,
$$

for $p_{k}(0)=p_{k}(1)=0$. The function $V_{0}(z, \tau)$ represents the transient component of the solution and decays exponentially with $\tau$. From this it is easy to show that, by introducing the intermediate variable $\tilde{\tau}=R t$, (10) matches to (7).

Note that this limit corresponds, physically, to a very slow rate of compression. In this situation we have found that the deformation is essentially uniform compaction of the solid matrix. Also, the transient effects are negligible, and the permeability of the tissue does not contribute until second order. However, the dependence of the surface stress on the diffusivity function $K$ is general and it is for this reason that this limit will be used later to determine $K$.

Moderate rates of compression. For the case of $R<<1$ the rate of compression is relatively fast, which means that by the end of the compressive stage the disturbance has not effectively reached the other end of the tissue. This semi-infinite nature of the material is evident in the singular nature of the limit. As should be expected, this is accounted for by introducing the coordinate

$$
\xi=z / R
$$

so that (4) becomes

$$
\partial_{\xi}^{2} u=K\left(\delta \partial_{\xi} u\right) \partial_{t} u
$$

where

$$
u(0, t)=g(t) \text { and } u(\xi, 0)=0
$$

Also,

$$
\delta=\varepsilon / R .
$$

For a slow rate of compression, as in the previous section, it is simply required that the equilibrium strain $\varepsilon$ is fixed and small. However, for the case at hand, it is necessary to specify how $\varepsilon$ compares with the rate constant $R$. The two cases that arise are when the permeability is a weakly nonlinear function, so $\delta$ is small, and when it is a strongly nonlinear function of the strain. The former case is simpler and leads to the strongly nonlinear case, so it is considered first. 
Assuming $\delta<<1$, the appropriate expansion of the displacement is

$$
u(\xi, t) \sim V_{0}(\xi, t)+\delta V_{1}+\ldots
$$

Also, it is assumed that the diffusive drag coefficient expands as follows:

$$
K(\delta) \sim 1-\delta M+\ldots,
$$

where $M$ is a positive constant. The first term in this expansion for $K$ is unity because of the normalization of $K^{*}$ in (5).

To determine the first two terms in (15) one must solve the following problems:

$O(1)$ :

$$
\partial_{\xi}^{2} V_{0}=\partial_{t} V_{0}
$$

where

$$
V_{0}(0, t)=g(t)
$$

and

$$
V_{0}(\infty, t)=V_{0}(\xi, 0)=0
$$

$O(\delta)$ :

$$
\partial_{\xi}^{2} V_{1}=\partial_{t} V_{1}-M \partial_{\xi} V_{0} \partial_{t} V_{0}
$$

where

$$
V_{1}(0, t)=V_{1}(\infty, t)=V_{1}(\xi, 0)=0 .
$$

Since these problems are linear it is possible to solve them in closed form, although the expressions are somewhat cumbersome. However, there is an important special case when the solution is relatively simple. If

$$
g(t)=t^{n} \quad \text { for } n \in \mathbb{Z}^{+},
$$

then the solution of (17) and (18) result in the following expansion:

$$
u(\xi, t) \sim t^{n} f_{0}(s)+\delta M t^{2 n-1 / 2} f_{1}(s)+\ldots,
$$

where $s$ is the similarity variable

$$
s=\xi / \sqrt{t} .
$$

The function $f_{0}$ is obtained from (17) and is given as

$$
f_{0}(s)=\left(1+s^{2} / 2\right) \operatorname{erfc}(s / 2)-s e^{-s^{2} / 4} / \sqrt{\pi}
$$

and $f_{1}$ satisfies the linear ordinary differential equation

$$
f_{1}^{\prime \prime}+s f_{1}^{\prime} / 2-3 f_{1} / 2=-f_{0}^{\prime} f_{0}^{\prime \prime}, \quad 0<s<\infty,
$$

for $f_{1}(0)=f_{1}(\infty)=0$. Therefore, the surface stress is

$$
\sigma_{0} \sim\left(\frac{2}{\sqrt{\pi}} t^{n-1 / 2}+\delta \alpha_{1} t^{2 n-1}+\ldots\right) / R,
$$


where $\alpha_{1}=-M f_{1}^{\prime}(0)$ is a positive constant. Also, note that since (17) is linear, (19) and (20) can be used to construct the solution for the case of when $g$ is a general analytic function.

These relatively simple expressions for the displacement and stress make it very easy to analyze the motion. For example, the material constants can be determined fairly quickly, in contrast to the earlier limit of a very slow rate of compression. It also hints at some problems that arise at the surface $z=0$, where the local strains can become significant. This problem becomes even more pronounced with the next limit.

Fast rates of compression. The remaining limit is the case when $R<<1$. As discussed earlier, this corresponds to a relatively fast rate of compression for a given equilibrium strain $\varepsilon$. This gives rise, as in the last section, to a spatial boundary layer that gradually diffuses into the tissue. However, rather than use (12), it is now necessary to consider other limits which correspond to how the time scale compares to $R$. To facilitate the analysis it is assumed that

$$
g(t)=t
$$

and, moreover,

$$
K(s)=1-M s,
$$

where $M$ is a positive constant.

The evolution of the deformation splits naturally into three subproblems, corresponding to whether the time scale is much less than, about the same size as, or much larger than the diffusion parameter $R$. In the initial moments the slope is small enough so that the tissue responds, essentially, as if the diffusion were linear. However, $\left|\varepsilon u_{z}\right|$ eventually becomes $O(1)$ so that both terms in the diffusive drag (23) are important. After this, $\left|\varepsilon u_{z}\right|$ becomes so large that the constant term in (23) can be ignored. These are the essential ideas of how the material responds; the details of the analysis follows.

To analyze the deformation in the initial moments the appropriate coordinates are

$$
\tilde{z}=z / R \sqrt{\zeta}, \quad \tilde{t}=t / \zeta
$$

and

$$
u=g(\zeta) v(\tilde{z}, \tilde{t}),
$$

where $\zeta$ is a function of $R$ to be determined. Introducing these into (4), one finds that

$$
\partial_{\tilde{z}}^{2} v=\left(1-\gamma \partial_{\tilde{z}} v\right) \partial_{t} v
$$

where

$$
v(0, \tilde{t})=g(\tilde{t})
$$

and

$$
\gamma=\varepsilon M g(\zeta) / R \sqrt{\zeta} .
$$

As we are considering the initial response, $\zeta$ is small compared to $R$. More precisely, it is 
assumed that $\gamma<<1$, that is

$$
\zeta<<(R / \varepsilon M)^{2} \text {. }
$$

In this case the appropriate expansion of $v(\tilde{z}, \tilde{t})$ is

$$
v \sim V_{0}(\tilde{z}, \tilde{t})+\gamma V_{1}(\tilde{z}, \tilde{t})+\ldots
$$

This gives rise to essentially the same problem as for the moderate rate of compression. Accordingly, $V_{0}$ satisfies (17) and $V_{1}$ satisfies (18). Thus,

$$
\begin{aligned}
& V_{0}=\tilde{t} f_{0}(\tilde{s}), \\
& V_{1}=\tilde{t}^{3 / 2} f_{1}(\tilde{s}),
\end{aligned}
$$

where

$$
\tilde{s}=\tilde{z} / \sqrt{\tilde{t}}
$$

The corresponding surface stress is given in (21).

The next limit is the distinguished one and arises when $\gamma=1$, that is, when

$$
\zeta=\left(R^{2} / \varepsilon M\right)^{z} .
$$

In this case (24) takes the form

$$
\partial_{\tilde{z}}^{2} v=\left(1-\partial_{\tilde{z}} v\right) \partial_{t} v
$$

where

$$
v(0, \tilde{t})=\tilde{t}
$$

Although it is difficult to say very much about the solution of this particular problem, it is possible, as shown below, to say how the solution emerges from this region. Before doing this, though, note that the particular form of $K(s)$ specified in (23) has not really been used, and so the analysis so far applies to any smooth function $K$ that satisfies (16). However, this is not true for the next limit.

The remaining case is when $\gamma>>1$, and so it is assumed that

$$
\zeta>>(R / \varepsilon M)^{2} \text {. }
$$

When this happens a spatial boundary layer appears, due to the nonlinearity, which is contained in the already established layer of thickness $R \sqrt{\zeta}$. To obtain the deformation in this new region set

$$
\bar{z}=\frac{z}{\eta}, \quad \bar{t}=\frac{1}{\zeta}\left(t-\frac{R^{2}}{\varepsilon^{2} M^{2}}\right)
$$

where

$$
\eta=R^{2} / \varepsilon M .
$$

Setting

$$
u(z, t)=g(\zeta) w(\bar{z}, \bar{t})
$$

we find

$$
\partial_{\bar{z}}^{2} w=\left(\beta-\partial_{\bar{z}} w\right) \partial_{t} w
$$


where

$$
\begin{aligned}
w(0, \bar{t}) & =\bar{t}+\beta, \\
w(\bar{z}, 0) & =\beta v(\bar{z}, 1), \\
\beta & =R^{2} / \varepsilon^{2} M^{2} \zeta .
\end{aligned}
$$

By assumption $\beta<<1$, and so, the appropriate expansion for $w$ is as follows:

$$
w \sim w_{0}(\bar{z}, \bar{t})+\beta w_{1}(\bar{z}, \bar{t})+\ldots
$$

Substituting this into (27) one finds that

$O(1)$ :

$$
\partial_{\bar{z}}^{2} w_{0}=-\partial_{\bar{z}} w_{0} \partial_{t} w_{0},
$$

where $w_{0}(0, \bar{t})=\bar{t}$, and $w_{0}(\infty, \bar{t})=w_{0}(\bar{z}, 0)=0$.

$O(\beta)$ :

$$
\partial_{\bar{z}}^{2} w_{1}=-\partial_{t} w_{0} \partial_{\bar{z}} w_{1}-\partial_{\bar{z}} w_{0} \partial_{t} w_{1}+\partial_{t} w_{0}
$$

where $w_{1}(0, \bar{t})=1$ and $w_{1}(\bar{z}, 0)=v(\bar{z}, 1)$. The $O(1)$ problem can be solved using similarity methods, from which it is found that

$$
w_{0}=\bar{t} f(\bar{z})
$$

where

$$
f^{\prime \prime}=-f^{\prime} f
$$

for $f(0)=1$ and $f(\infty)=0$. Thus,

$$
f(\bar{z})=2 /(2+\bar{z}) .
$$

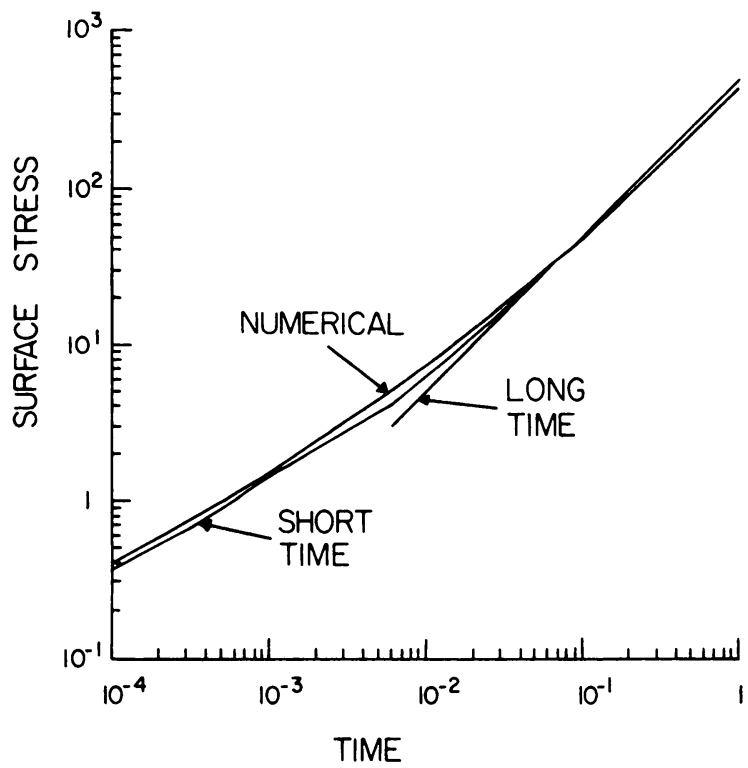

Fig. 1. Surface stress (6) as found from (4), using the Crank-Nicolson scheme and the $K(s)$ given in (23), as well as the short- and long-time asymptotic approximations given in (21) and (31), respectively. In this particular case, $R^{2}=10^{-3}$ and $\varepsilon M=1.0$. 
The $O(\beta)$ problem is linear, although it has variable coefficients. In solving it, it is necessary to match $w_{1}$ with the solution in the region $z \sim R \sqrt{t}$. This outer region arises from the evolution of the problem considered in the last paragraph, however the displacement is now $O(R \sqrt{t} / \varepsilon M)$. Also note that if the forcing function given in (19) were used rather than (22), then (28) would be replaced with $\bar{t}^{n} F\left(z t^{n-1}\right)$ and there would be a corresponding change in (29). In any case, with the above solution the surface stress (6) is given to first order as

$$
\sigma_{0} \sim \varepsilon M t / 2 R^{2} .
$$

It should be remembered that this applies only after $t$ is at least $O\left(R^{2} / \varepsilon^{2} M^{2}\right)$.

To illustrate the contribution from these three temporal regions, (21) and (31) are plotted in Fig. 1 for the case of when $R^{2}=10^{-3}$ and $\varepsilon M=1$. Also shown is the corresponding numerical solution of (4) using the Crank-Nicolson scheme. It is seen from this figure that, in their respective regions, the asymptotic results are good approximations to the solution and at the same time they are relatively simple analytically.

Applications. For articular cartilage, the time scales involved with the moderate and fast rates of compression arise the most frequently. However, slow rates do occur and are definitely obtainable experimentally. As pointed out earlier, these very slow rates have the advantage of enabling us to determine the diffusive drag function up to a given equilibrium strain. Alternatively, if one of the various pore models are used to obtain the functional form of $K(s)$, then this limit can be used to analyze the various components of the microstructure, such as the porosity.

To illustrate these ideas, consider the case when $g(t)=t$ so the surface stress (8) takes the form

$$
\sigma_{0}(t) \sim t+K(-\varepsilon t) / 3 R^{2} \text { for } 0<t \leqslant 1 .
$$

Given the empirical determination of $\sigma_{0}$, it follows that

$$
K(s)=3 R^{2}\left[s+\varepsilon \sigma_{0}(-s / \varepsilon)\right] / \varepsilon .
$$

In using this expression to determine $K$ it should be remembered that (8) does not apply for small $t$; in particular, it does not apply for $t$ up to $O\left(1 / R^{2}\right)$. Also, (32) is only an asymptotic approximation, and so it contains a certain error. With the two-term expansion used here the error is $O\left[K(-\varepsilon) K^{\prime}(-\varepsilon) / R^{4}\right]$.

In the experimental investigation of the compressive properties of cartilage it is found that the stress, for a slow rate, has the history illustrated in Fig. 2 [4]. Using the near linearity of this result and (32), we find that

$$
K(s)=1-\left(\frac{\sigma_{p}-\sigma_{L}-1}{\varepsilon \sigma_{L}}\right) s,
$$

where $\sigma_{L}$ represents the linear extrapolation of the curve to $t=0$. From (2) it follows that

$$
k^{*}\left(\partial_{z^{*}} u^{*}\right)=k_{0} /\left(1-M \partial_{z^{*}} u^{*}\right),
$$

where $k_{0}$ and $M$ are material constants. In fact, from (33) and (5) we get 


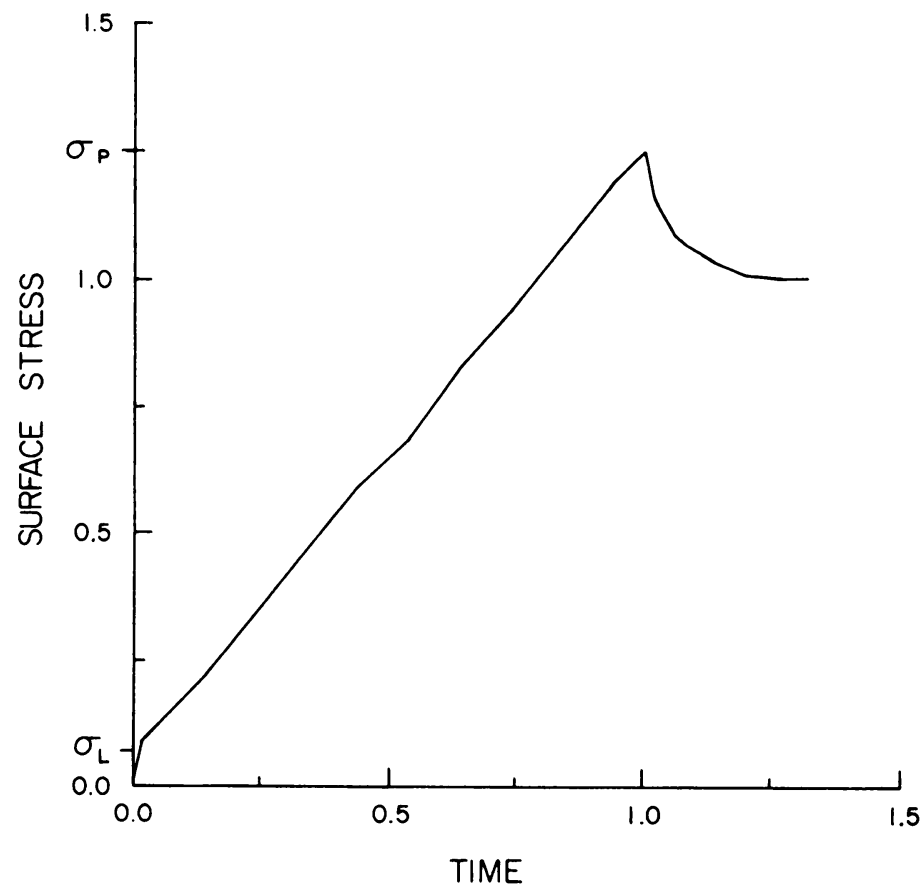

FIG. 2. The normalized surface stress obtained in a typical slow applied compression test for bovine articular cartilage. In this case, $\varepsilon=5 \%, t_{0}=3000 \mathrm{~s}$., $h=1.6 \times 10^{-3} \mathrm{~m}$, and $\sigma_{\infty}^{*}=0.3$ MPA.

$$
k_{0}=\left(\phi^{f}\right)^{2} / K^{*}(0)=-\varepsilon h^{2} / 3 t_{0} \sigma_{L}^{*}
$$

and

$$
M=\left(\sigma_{p}-\sigma_{L}-1\right) / \varepsilon \sigma_{L} .
$$

Note that $k_{0}$ in (35) is positive since $\sigma_{L}^{*}$ is negative and $\varepsilon$ is positive.

Using the first principal invariant $I^{s}$ of the infinitesimal strain tensor for the deformation of the elastic solid, (34) can be written as

$$
K^{*}\left(I^{s}\right)=k_{0} /\left(1-M I^{s}\right) .
$$

The intrinsic permeability function obtained by Lai and Mow [3] from their permeation experiments is

$$
k^{*}\left(I^{s}\right)=k_{0} e^{M I^{s}} .
$$

Comparing this with (36) and recalling the small-strain assumption made earlier, it is seen that they agree to second order in $I^{s}$, which is consistent with the degree of approximation used to obtain (36).

Another application of the analysis concerns the determination of the consistency of the original modeling assumptions. For a fast rate of compression and a permeability function as given in (36) the strain at the surface $z=0$ is $-\varepsilon^{2} M^{2} t / 2 R^{2}$. Since $\varepsilon^{2} M^{2} / R^{2}>>1$, by the end of the compressive stages $(t=1)$ the strain is considerable. This indicates that the 
original assumption of a linear stress-strain relationship is doubtful in this range. Also note that this is true even though the eventual equilibrium strain $\varepsilon$ is relatively small. In any event, the solution should give a qualitative indication of the response. However, it also shows that for a realistic model of cartilage it is going to be necessary to account for the nonlinear nature of the material, particularly in the region near the articular surface.

\section{REFERENCES}

[1] R. M. Bowen, Theory of mixtures, in Continuum physics (A. E. Erigen, ed.), Academic Press, New York, 1976, pp. $1-127$

[2] R. E. Craine, A. E. Green, and P. M. Naghdi, A mixture of viscous elastic materials with different constituent temperatures, Q. J. Appl. Math. 23, 171-184 (1970)

[3] W. M. Lai and V. C. Mow, Drag-induced compression of articular cartilage during a permeation experiment, Biorheology 17, 110-123 (1980)

[4] W. M. Lai, V. C. Mow, and V. Roth, Effects of a nonlinear strain-dependent permeability and rate of compression on the stress behavior of articular cartilage, J. Biomech. Eng. 103K, 61-66 (1981)

[5] V. C. Mow, S. C. Kuei, W. M. Lai, and C. G. Armstrong, Biphasic creep and stress relaxation of articular cartilage: theory and experiments, J. Biomech. Eng. 102K, 73-84 (1980)

[6] J. J. Shi, K. K. Rajagopal, and A. S. Wineman, Application of the theory of interacting continua to the diffusion of a fluid through a non-linear elastic media, Int. J. Eng. Sci. 19, 871-889 (1981) 